\title{
Superior temporal gyrus volume in antipsychotic-naive people at risk of psychosis
}

Tsutomu Takahashi, Stephen J. Wood, Alison R. Yung, Mark Walterfang, Lisa J. Phillips, Bridget Soulsby, Yasuhiro Kawasaki, Patrick D. McGorry, Michio Suzuki, Dennis Velakoulis and Christos Pantelis

\section{Background}

Morphological abnormalities of the superior temporal gyrus have been consistently reported in schizophrenia, but the timing of their occurrence remains unclear.

\section{Aims \\ To determine whether individuals exhibit superior temporal gyral changes before the onset of psychosis.}

\section{Method}

We used magnetic resonance imaging to examine grey matter volumes of the superior temporal gyrus and its subregions (planum polare, Heschl's gyrus, planum temporale, and rostral and caudal regions) in 97

antipsychotic-naive individuals at ultra-high risk of psychosis of whom 31 subsequently developed psychosis and 66 did not, and 42 controls.

\section{Results}

Those at risk of psychosis had significantly smaller superior temporal gyri at baseline compared with controls bilaterally, without any prominent subregional effect; however, there was no difference between those who did and did not subsequently develop psychosis.

\section{Conclusions}

Our findings indicate that grey matter reductions of the superior temporal gyrus are present before psychosis onset, and are not due to medication, but these baseline changes are not predictive of transition to psychosis.

\section{Declaration of interest}

None.
Morphological abnormalities of the superior temporal gyrus, ${ }^{1-3}$ and its functionally relevant subregions such as Heschl's gyrus and planum temporale, ${ }^{4,5}$ in schizophrenia appear to be already present at onset of overt psychosis, but the time course of their occurrence remains unclear. Magnetic resonance imaging (MRI) studies have demonstrated that among participants at ultra-high risk of psychosis, ${ }^{6,7}$ those who subsequently developed psychosis had less grey matter than those who did not in frontotemporolimbic-paralimbic regions, including the right anterior part of the superior temporal gyrus, prior to psychosis onset. ${ }^{8,9}$ However, the conclusions that can be drawn from these analyses are limited by the potential methodological problems of voxel-based morphometry (VBM) ${ }^{10}$ and the inclusion of people taking medication such as antipsychotics or antidepressants. One MRI study investigating regions of interest in individuals at high genetic risk demonstrated smaller superior temporal gyral volumes bilaterally compared with controls, ${ }^{11}$ but this study did not examine diagnostic outcome (i.e. later transition) and subregional specificity of the superior temporal gyrus. Our longitudinal region-of-interest study did not detect baseline superior temporal gyrus changes in the individuals who went on to develop psychosis, ${ }^{12}$ but was limited by a small sample size. We sought to address these limitations by conducting a regionof-interest analysis of the superior temporal gyrus subregions in a relatively large sample of antipsychotic-naive individuals at ultra-high risk who did and did not later develop a psychotic disorder, and healthy controls. Based on previous work, ${ }^{8,9}$ we predicted that the high-risk group who developed psychosis would have a smaller superior temporal gyrus at baseline compared with controls.

\section{Method}

\section{Participants}

Antipsychotic-naive individuals at ultra-high risk of psychosis ( $n=97)$ were recruited between 1995 and 2001 from admissions to the Personal Assessment and Crisis Evaluation clinic, Melbourne, Australia, which was established to identify young people at clinical risk of developing a first psychotic episode within a short follow-up period. ${ }^{7}$ Those recruited were aged 1430 years, had not experienced a previous psychotic episode, had never received antipsychotic medication (antipsychotics, antidepressants, mood stabilisers or benzodiazepines) and had an IQ score above 70, assessed with the National Adult Reading Test. ${ }^{13}$ At intake, the Comprehensive Assessment of At Risk Mental States (CAARMS), ${ }^{14}$ a structured clinical interview designed to assess prodromal symptoms and risk of psychosis, was administered. General psychopathology was assessed using the Brief Psychiatric Rating Scale (BPRS), ${ }^{15}$ negative symptoms were rated with the Scale for the Assessment of Negative Symptoms (SANS), ${ }^{16}$ and family history of psychosis in a firstor second-degree relative was assessed using the Family Interview for Genetic Studies, ${ }^{17}$ as well as interviews with a family member. The CAARMS-defined risk identification criteria and their rationale have been fully described elsewhere. ${ }^{18}$ Briefly, individuals considered to be at ultra-high risk are characterised by one or more of the following:

(a) attenuated psychotic symptoms, defined by subthreshold intensity or frequency;

(b) brief limited intermittent psychotic symptoms with spontaneous resolution within 1 week;

(c) family history of psychosis (first-degree relative) or a personal history of schizotypal personality disorder, accompanied by a decline in general functioning.

The group at risk were monitored regularly over a minimum 12-month period (mean 1.1 years, maximum 3.7 years) for the onset of full-blown psychosis and were then divided into subgroups based on operationalised criteria for psychosis onset using CAARMS and the Structured Clinical Interview for DSM-IV Axis I disorders. ${ }^{19}$ In total, 31 (32\%) of this ultra-high 
risk group developed a psychotic illness (UHR-P) and $66(68 \%)$ did not (UHR-NP) during follow-up. Of those who developed psychosis, 19 developed schizophrenia-spectrum disorder (17 schizophrenia, 2 schizoaffective disorder), 10 an affective psychosis (5 major depressive disorder with psychotic features, 5 bipolar disorder with psychotic features) and 2 other psychoses ( 1 psychosis not otherwise specified, 1 brief psychosis). Fifty of the at-risk sample had a positive family history of psychosis.

Healthy volunteers $(n=42)$ with sociodemographic characteristics similar to the high-risk group were recruited by approaching ancillary hospital staff and through advertisements; those with a personal or family history of psychiatric illness were excluded. All participants were screened for comorbid medical and psychiatric conditions by clinical assessment and by physical and neurological examination. Exclusion criteria were a history of significant head injury, seizures, neurological disease, impaired thyroid function, diabetes, corticosteroid use, or alcohol or substance misuse or dependence meeting DSM-IV criteria. ${ }^{20}$ Of the 97 at-risk participants in this study, 64 (22 UHR-P and 42 UHR-NP) had taken part in our earlier VBM study, ${ }^{8}$ and 31 (11 UHR-P and 20 UHR-NP) had taken part in our longitudinal superior temporal gyrus study. ${ }^{12}$ The study reported here was approved by local research and ethics committees, and written informed consent was obtained from the participants or their parents/guardians where appropriate.

\section{Imaging procedure}

Magnetic resonance scans were acquired with a $1.5 \mathrm{~T}$ GE Signa scanner (General Electric Medical Systems, Milwaukee, Wisconsin, USA). A three-dimensional volumetric spoiled gradient recalled echo sequence generated 124 contiguous $1.5 \mathrm{~mm}$ coronal slices (repetition time $(\mathrm{TR})=14.3 \mathrm{~ms}$, time to echo $(\mathrm{TE})=3.3 \mathrm{~ms}$, flip angle $=30^{\circ}$, field of view $=24 \mathrm{~cm} \times 24 \mathrm{~cm}$, matrix $=256 \times 256$, voxel dimensions $=0.938 \mathrm{~mm} \times 0.938 \mathrm{~mm} \times 1.5 \mathrm{~mm}$ ). The scanner was calibrated fortnightly with the same phantom to ensure stability of measurements.

On a Unix workstation the image data were coded randomly and analysed with the software package Dr View version 5 (AJS, Tokyo, Japan). Brain images were realigned in three dimensions and reconstructed into contiguous coronal images, with a $0.938 \mathrm{~mm}$ thickness, perpendicular to the anterior-posterior commissural line. The whole cerebrum was manually separated from the brain stem and cerebellum. The signal-intensity histogram distributions from the $T_{1}$-weighted images across the whole cerebrum were used to segment the voxels semi-automatically into grey matter, white matter and cerebrospinal fluid. The intracranial volume was measured to correct for differences in head size as previously described. ${ }^{21}$

\section{Volumetric analyses of superior temporal subregions}

The grey matter of the superior temporal gyrus subregions (planum polare, Heschl's gyrus, planum temporale, rostral and caudal superior temporal gyrus; online Fig. DS1) was manually traced on $0.938 \mathrm{~mm}$ consecutive coronal slices as described in detail elsewhere. ${ }^{12,22}$ Briefly, the first coronal plane showing the temporofrontal junction and the coronal plane containing the posterior end of the posterior horizontal limb of the sylvian fissure were chosen as the anterior and posterior boundaries respectively of the whole superior temporal gyrus. On each coronal slice the whole superior temporal gyrus was bounded superiorly by the sylvian fissure and inferiorly by the superior temporal sulcus. The gyrus was then segmented into supratemporal and lateral portions by the lateral limb of the supratemporal plane. Heschl's gyrus was traced from posterior to anterior, beginning with the first slice containing Heschl's sulcus and ending anteriorly with the slice containing the most anterior point of Heschl's sulcus or the sulcus intermedius if it existed. On each coronal slice, Heschl's gyrus was bounded medially by the sylvian fissure, inferior circular insular sulcus or the first transverse sulcus and laterally by Heschl's sulcus. After tracing the Heschl's gyrus that takes a diagonal course on the supratemporal plane, the regions lying anteromedial and posterolateral to the gyrus within the remaining grey matter of the supratemporal plane were regarded as planum polare and planum temporale respectively. The lateral superior temporal gyrus was divided into rostral and caudal portions by the plane including the anterior tip of Heschl's gyrus.

All volumetric data reported here were measured by one rater (T.T.) masked to the participants' identities. Intra-rater (T.T.) and interrater (T.T., Y.K.) intraclass correlation coefficients in eight randomly selected brains exceeded 0.88 for all subregions.

\section{Statistical analysis}

Clinical and demographic differences between groups were examined with one-way analysis of variance (ANOVA) or chisquared tests. The relative volume of the superior temporal gyrus, calculated as (absolute volume/intracranial volume) $\times 100$, was assessed using a repeated-measures analysis of covariance (ANCOVA) with age as a covariate, with group (control, UHR-NP and UHR-P) and gender as between-participant factors, and subregion (planum polare, Heschl's gyrus, planum temporale, rostral superior temporal gyrus, caudal superior temporal gyrus) and side (left, right) as within-participant variables. For the at-risk group, the effects of family history ( $n=50$ with history $v . n=47$ without history) and type of psychosis developed (schizophrenia-spectrum disorder $n=19 \mathrm{v}$. affective psychosis $n=10$ ) on relative superior temporal gyrus volume were also examined by ANCOVA. The post hoc Tukey's test was employed to follow up the significant main effects or interactions. We performed a Cox regression analysis to examine whether superior temporal gyrus volume would predict later transition, using transition to psychosis as the status variable, time to onset (UHR-P) or follow-up period (UHR-NP) as the time variable, and relative superior temporal gyrus volume and intake BPRS and SANS scores as covariates. The relationships between the relative superior temporal gyrus volumes and demographic or clinical variables were examined with Pearson's $r$ (time between scan and psychosis onset was log-transformed because of skewed distributions). Owing to the lack of prominent subregional effects, we used the whole superior temporal gyrus volume for the regression and correlational analyses. Statistical significance was defined as $P<0.05$ (two-tailed).

\section{Results}

Comparison of the control, UHR-P and UHR-NP groups revealed no significant difference in age, gender, handedness or IQ score (Table 1). Participants in the control group were taller than those in the UHR-NP group, but the groups did not differ significantly in their intracranial volume. Baseline SANS score, but not BPRS score, was higher in the UHR-P group.

\section{Superior temporal gyrus volumes}

Analysis of covariance of the relative superior temporal gyral volumes revealed significant main effects for group $\left(F_{(2,132)}=3.36\right.$, $P=0.038)$, side $\quad\left(F_{(1,133)}=99.13, \quad P<0.001\right)$ and subregion $\left(F_{(4,532)}=599.56, P<0.001\right)$, and a significant side $\times$ subregion interaction $\left(F_{(4,532)}=14.54, P<0.001\right)$. There was no group $\times$ 


\begin{tabular}{|c|c|c|c|c|}
\hline & Controls $(n=42)$ & UHR-NP $(n=66)$ & UHR-P $(n=31)$ & Group comparisons \\
\hline Age, years: mean (s.d.) & $20.0(2.8)$ & $20.2(3.3)$ & $19.1(3.6)$ & $F(2,136)=1.34, P=0.265$ \\
\hline Male/female, $n / n$ & $28 / 14$ & $39 / 27$ & 20/11 & $\chi^{2}=0.69, P=0.71$ \\
\hline Handedness, $n$ (right/mixed/left) ${ }^{\mathrm{a}}$ & $38 / 1 / 2$ & $54 / 2 / 9$ & $28 / 0 / 3$ & $P=0.52$, Fisher's exact test \\
\hline Height, cm: mean (s.d.) $)^{a}$ & $175.7(9.7)$ & $171.2(8.5)$ & $172.3(8.9)$ & $F(2,132)=3.20, P=0.044 ;$ controls $>$ UHR-NP \\
\hline Premorbid IQ, mean (s.d.) ${ }^{\mathrm{a}}$ & $97.9(8.9)$ & 95.7 (13.5) & 93.9 (13.6) & $F(2,118)=0.77, P=0.464$ \\
\hline \multicolumn{5}{|l|}{ Time between scan and onset, days } \\
\hline Mean (s.d.) & - & - & 199 (199) & - \\
\hline Median & - & - & 104 & - \\
\hline BPRS score at intake, mean (s.d.) & - & $17.9(7.1)$ & $18.9(7.2)$ & $F(1,95)=0.36, P=0.551$ \\
\hline SANS score at intake, mean (s.d.) & - & $19.7(14.2)$ & $29.0(15.7)$ & $F(1,95)=8.37, P=0.005 ;$ UHR-P $>$ UHR-NP \\
\hline Intracranial volume, $\mathrm{cm}^{3}$ : mean (s.d.) & $1453(152)$ & $1416(146)$ & $1470(129)$ & $F(2,144)=1.86, P=0.160^{b}$ \\
\hline \multicolumn{5}{|c|}{$\begin{array}{l}\text { BPRS, Brief Psychiatric Rating Scale; SANS, Scale for the Assessment of Negative Symptoms; UHR-NP, ultra-high risk group who did not develop psychosis; UHR-P, ultra-high } \\
\text { risk group who did develop psychosis. } \\
\text { a. Data missing for some participants. } \\
\text { b. Analysis of covariance with age as covariate and group as between-participant factor. }\end{array}$} \\
\hline
\end{tabular}

subregion $\left(F_{(8,532)}=1.07, P=0.384\right)$ or group $\times$ side $\times$ subregion $\left(F_{(8,532)}=0.54, P=0.821\right)$ interaction, implying that group difference in superior temporal gyrus volume was not highly localised to a particular subregion. These ANCOVA results remained the same when we analysed the absolute superior temporal gyrus volume with intracranial volume and age as covariates.

Post hoc tests showed that the superior temporal gyral volume was significantly smaller in the UHR-P group compared with the control group $(P=0.031)$, but there was no difference between the UHR-NP and control groups $(P=0.133)$, or the UHR-NP and UHR-P groups $(P=0.576)$. Heschl's gyrus $(P<0.001)$ and planum temporale $(P<0.001)$ had a significant asymmetrical pattern (left $>$ right) for all groups. No group difference was found for any superior temporal gyrus subregion (Table 2, Fig. 1). When the UHR-P and UHR-NP groups were categorised together, the group as a whole had a significantly smaller whole superior temporal gyrus compared with controls bilaterally (group effect, $F_{(1,134)}=5.48, P=0.021$; post hoc test, $\left.P=0.014\right)$.

No difference in superior temporal gyral volume was found between the at-risk participants with and without a family history of psychosis $\left(F_{(1,94)}=1.00, P=0.319\right)$, or between those in the UHR-P group who later developed schizophrenia-spectrum disorder $(n=19)$ and those who developed affective psychosis $\left(n=10 ; F_{(1,26)}=0.61, P=0.443\right)$.

\section{Regression and correlational analyses}

The SANS ratings predicted time to transition (Wald test $=5.784$, $\beta=1.034,95 \%$ CI $1.01-1.06, P=0.016)$, but the superior temporal gyrus volume (left, $P=0.123$; right, $P=0.658$ ) and BPRS score $(P=0.819)$ were not predictive of later transition. The relative whole superior temporal gyrus volumes were not correlated with height and IQ score for all groups. There was a negative correlation between age and right superior temporal gyral volume only for the UHR-P group $(r=-0.574, P<0.001)$; this correlation was significantly different from that in the other groups $(v$. UHR-NP, $P=0.010 ; v$. controls, $P=0.020$ ) (Fig. 2). Correlational analyses did not reveal any significant correlation between the superior temporal gyral volumes and intake BPRS and SANS scores in either UHR group ( $r$ values -0.033 to 0.109 , all $P>0.388$ ) or time to the psychosis onset $(\log )$ in the UHR-P group.

\section{Discussion}

Compared with healthy controls, the ultra-high risk cohort, especially the UHR-P group, showed diffuse (not highly localised to a particular subregion) grey-matter reductions of bilateral superior temporal gyrus, whereas there was no difference in superior temporal gyral volumes between the UHR-NP and control or UHR-P groups. We also demonstrated that the superior temporal gyral volume did not predict who would develop psychosis.

\section{Changes in superior temporal gyrus as a vulnerability marker}

Consistent with previous VBM studies, ${ }^{8,9}$ our results suggest that the superior temporal gyrus changes in individuals at ultra-high risk of psychosis who subsequently develop full-blown psychosis are present before illness onset. Our findings indicate that these changes are not due to the effect of medication. The UHR-NP group also exhibited non-significant but mild grey matter reductions relative to the control group (effect size $>0.2$ ), especially in left posterior regions (Heschl's gyrus, planum temporale and caudal superior temporal gyrus). Furthermore, the ultra-high risk group as a whole (regardless of later transition) demonstrated significant grey matter reduction of the bilateral superior temporal gyrus compared with controls, consistent with previous MRI studies in clinically or genetically predisposed highrisk individuals. ${ }^{9,11,23}$ These findings are in line with the fact that those in the at-risk group who did not progress to psychosis were not asymptomatic, as evidenced by the range of non-psychotic diagnoses, ${ }^{6,7}$ and also had experienced subthreshold psychotic symptoms, supporting the hypothesis that such superior temporal gyrus changes represent a vulnerability marker. ${ }^{9}$ However, given the issue of region-of-interest definition in this study (e.g. an external landmark for the anterior boundary of the superior temporal gyrus), ${ }^{12}$ the possibility also exists that anterior reductions in the superior temporal gyrus are more prominent in the individuals with future transition. ${ }^{8,9}$

\section{Onset-related superior temporal gyrus change}

These cross-sectional findings are in line with our recent longitudinal region of interest analysis in a small ultra-high risk sample, ${ }^{12}$ which demonstrated progressive grey matter reduction of the superior temporal gyrus in the UHR-P group during the transition phase (left $-5.0 \%$ per year, right $-3.9 \%$ per year). The current UHR-P cohort exhibited lesser reductions in superior temporal gyral grey matter (left $-9.8 \%$, right $-2.6 \%$ ) relative to controls compared with those in our previous region-of-interest study in first-episode schizophrenia (left $-16.4 \%$, right 


\begin{tabular}{|c|c|c|c|c|c|c|c|c|c|}
\hline & \multirow{2}{*}{\multicolumn{3}{|c|}{ Grey matter volume, mm³: mean (s.d.) }} & \multicolumn{6}{|c|}{ Analysis of covariance ${ }^{a, b}$} \\
\hline & & & & \multicolumn{2}{|c|}{ Group } & \multicolumn{2}{|c|}{ Side } & \multicolumn{2}{|c|}{ Group $\times$ side } \\
\hline & $\begin{array}{l}\text { Controls } \\
(n=42)\end{array}$ & $\begin{array}{l}\text { UHR-NP } \\
(n=66)\end{array}$ & $\begin{array}{l}\text { UHR-P } \\
(n=31)\end{array}$ & $F(2,135)$ & $P$ & $F(1,136)$ & $P$ & $F(2,136)$ & $P$ \\
\hline $\begin{array}{l}\text { Whole STG } \\
\text { Left } \\
\text { Right }\end{array}$ & $\begin{array}{l}15053(2582) \\
12746(1959)\end{array}$ & $\begin{array}{l}13903 \text { (2599) } \\
12183(2181)\end{array}$ & $\begin{array}{l}13573^{\mathrm{C}}(1875) \\
12417^{\mathrm{C}}(1776)\end{array}$ & 3.87 & 0.023 & 118.65 & $<0.001$ & 3.11 & 0.048 \\
\hline $\begin{array}{l}\text { Planum polare } \\
\text { Left } \\
\text { Right }\end{array}$ & $\begin{array}{l}1984(466) \\
1804(508)\end{array}$ & $\begin{array}{l}1969(615) \\
1754(573) \\
\end{array}$ & $\begin{array}{l}1912(428) \\
1721(445) \\
\end{array}$ & 0.63 & 0.533 & 18.05 & $<0.001$ & 0.13 & 0.883 \\
\hline $\begin{array}{l}\text { Heschl's gyrus } \\
\text { Left } \\
\text { Right }\end{array}$ & $\begin{array}{l}2609(842) \\
1846(474) \\
\end{array}$ & $\begin{array}{l}2282(624) \\
1759(490)\end{array}$ & $\begin{array}{l}2293(806) \\
1921(534) \\
\end{array}$ & 1.12 & 0.331 & 99.86 & $<0.001$ & 2.82 & 0.063 \\
\hline $\begin{array}{l}\text { Planum temporale } \\
\text { Left } \\
\text { Right }\end{array}$ & $\begin{array}{l}3772(837) \\
2875(671) \\
\end{array}$ & $\begin{array}{l}3393(864) \\
2756(712)\end{array}$ & $\begin{array}{l}3351(818) \\
2851(667)\end{array}$ & 2.20 & 0.115 & 103.70 & $<0.001$ & 2.34 & 0.100 \\
\hline $\begin{array}{l}\text { Rostral STG } \\
\text { Left } \\
\text { Right }\end{array}$ & $\begin{array}{l}1598(636) \\
1421(632) \\
\end{array}$ & $\begin{array}{l}1718(781) \\
1490(700)\end{array}$ & $\begin{array}{l}1446(480) \\
1349(591) \\
\end{array}$ & 2.13 & 0.122 & 7.39 & 0.007 & 0.40 & 0.669 \\
\hline $\begin{array}{l}\text { Caudal STG } \\
\text { Left } \\
\text { Right }\end{array}$ & $\begin{array}{l}5054(1124) \\
4760(1050)\end{array}$ & $\begin{array}{l}4507 \text { (1061) } \\
4394(1001)\end{array}$ & $\begin{array}{l}4523(976) \\
4601(1027)\end{array}$ & 3.34 & 0.038 & 1.26 & 0.264 & 0.798 & 0.452 \\
\hline
\end{tabular}

$-15.5 \%) .^{24}$ The results of a cross-sectional VBM study by Borgwardt et al were similar to ours: the superior temporal gyrus in high-risk participants with later transition was significantly larger than that in first-episode psychosis, but was smaller (at a less stringent statistical threshold) in the right hemisphere than in controls. ${ }^{25}$ Taken together, these superior temporal gyrus observations support the model of psychosis that morphological abnormalities seen in patients reflect a combination of pre-existing vulnerability and changes associated with the first expression of psychotic symptoms. ${ }^{26}$

We found no effect of family history of psychosis on superior temporal gyrus volume in our at-risk sample, consistent with a previous negative finding for this region in a large genetically high-risk sample $(n=146){ }^{27}$ These findings suggest that the superior temporal gyral volume changes related to psychosis are more likely to be acquired, e.g. due to environmental influences later in development, ${ }^{26,28}$ than genetic in origin. A significant effect of age on superior temporal gyrus volume only in the UHR-P group may be consistent with the notion that the transition to psychosis is associated with an acceleration of normal brain maturational processes. ${ }^{27,28}$

\section{Clinical implications}

Given the role of the superior temporal gyrus in manifesting psychotic symptoms such as hallucinations or thought disorder, ${ }^{29,30}$ as well as relevance to social cognitive function, ${ }^{31}$ pre-onset superior temporal gyral changes might at least partly underpin prodromal symptoms such as subthreshold psychotic symptoms or increasing social withdrawal, ${ }^{7}$ although we found no correlation

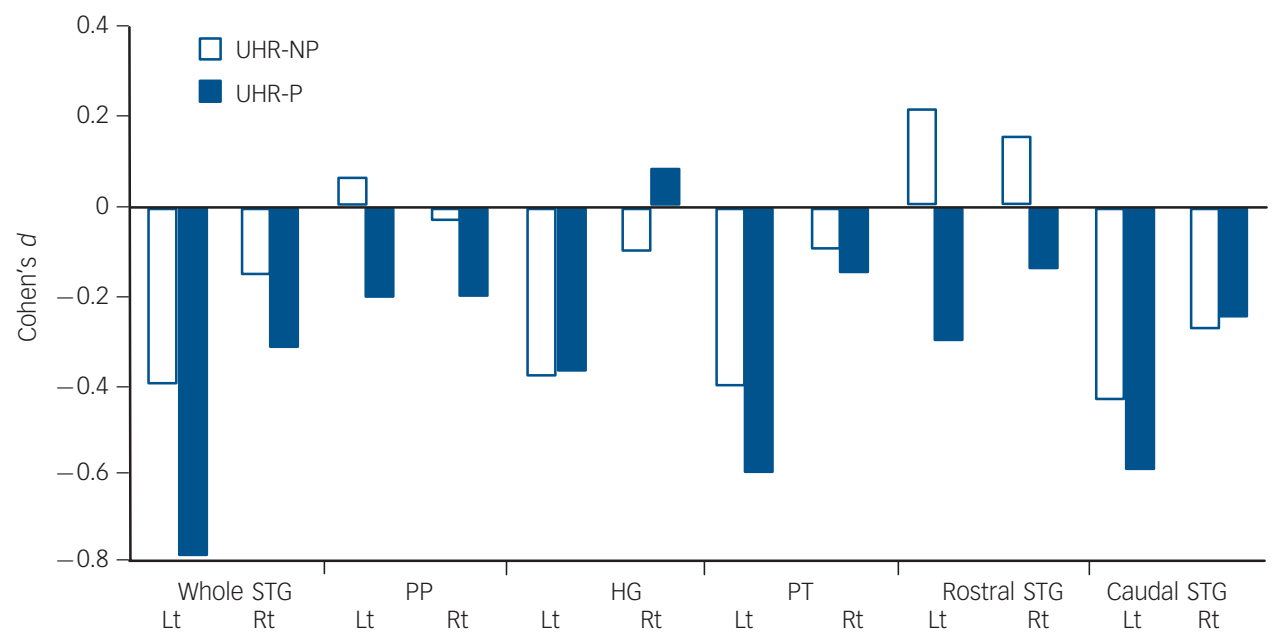

Fig. 1 Effect sizes (Cohen's d) based on relative superior temporal gyrus (STG) volumes for ultra-high risk participants who did (UHR-P) and did not (UHR-NP) develop psychosis relative to healthy controls. HG, Heschl's gyrus; PP, planum polare; PT, planum temporale. 
(a)

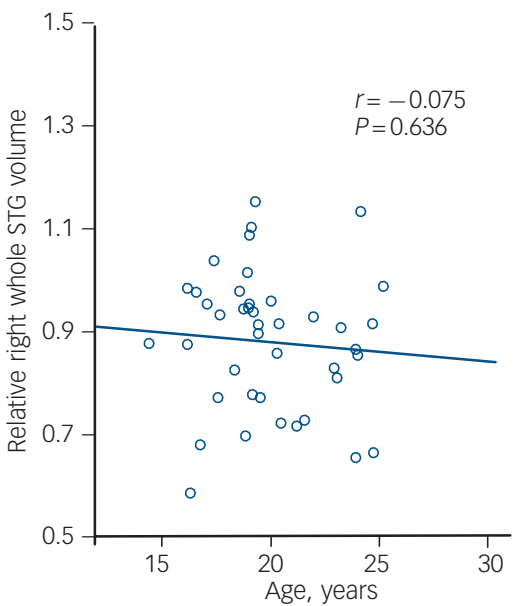

(b)

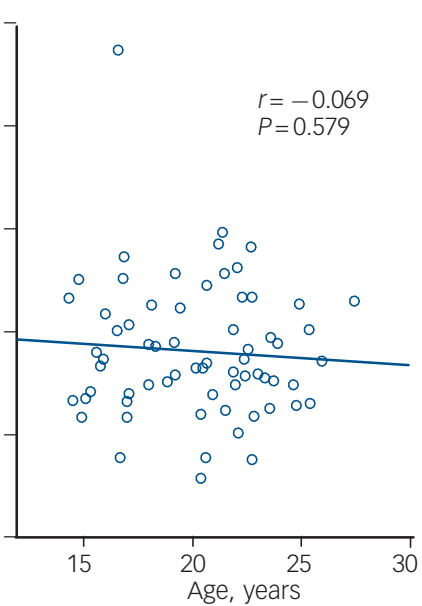

(c)

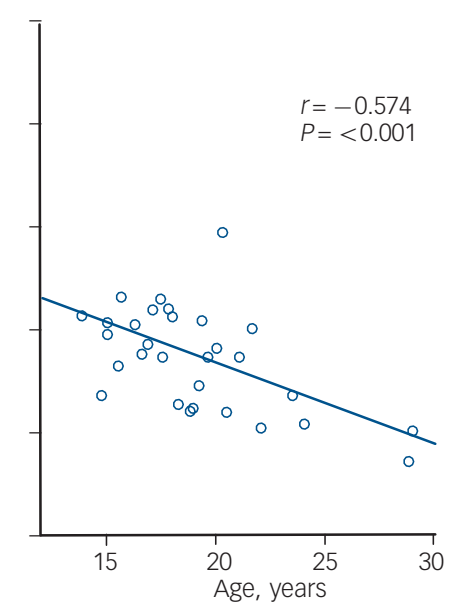

Fig. 2 Correlations between age and relative volumes of the right whole superior temporal gyrus (STG) in a healthy control group (a) and in individuals at ultra-high risk who did not (b) and did (c) later develop psychosis.

between superior temporal gyrus volumes and symptom measures in our ultra-high risk group. However, our own work has demonstrated a significant relationship between ongoing superior temporal gyrus reduction and subsequent positive symptoms in people with first-episode psychosis. ${ }^{12}$ These findings suggest that it is the progressive superior temporal gyrus changes during early phases of the illness that are relevant to clinical manifestations of psychosis.

One major aim of high-risk group studies has been to identify neurobiological and clinical predictors of future transition to psychosis, such as high level of depression, poor functioning and negative symptoms, ${ }^{7}$ which would allow specific and targeted preventive strategies. ${ }^{32} \mathrm{~A}$ series of our MRI studies has identified such predictive markers, including pituitary volume, thickness of the anterior cingulate cortex and corpus callosum shape. ${ }^{3-35}$ The study reported here replicated the predictive validity of the SANS score, $^{7}$ but did not support the role of pre-existing superior temporal gyrus volume reduction as a significant predictor of future transition. However, the onset-related ongoing superior temporal gyral changes, which could affect subsequent course of the illness, ${ }^{12}$ and potential ameliorating effects of antipsychotics, ${ }^{36}$ may implicate the potential role of the superior temporal gyrus changes as a therapeutic target during the early phases or an outcome marker after the onset of psychosis.

\section{Limitations}

Some limitations of this study should be taken into account. First, although all participants tested were antipsychotic-naive at the time of scanning, 11 individuals (UHR-P $n=3$, UHR-NP $n=6$, control $n=2$ ) had records of a significant degree of cannabis use, which could affect brain morphology. ${ }^{37}$ However, exclusion of these participants did not change the statistical conclusions. Second, the superior temporal gyrus findings in the ultra-high risk group are partly inconsistent with our previous investigations despite considerable sample overlap. Our VBM study found smaller superior temporal gyral volumes in the UHR-P group only for the right hemisphere, ${ }^{8}$ but it should be noted that this VBM study used $T_{2}$-weighted and proton density images in $3 \mathrm{~mm}$ thick slices that might hinder detection of subtle changes, and that the use of VBM has been criticised because of its inadequacy in dealing with problems of brain registration. ${ }^{10}$ Although our longitudinal region-based analysis failed to detect baseline superior temporal gyrus changes in the at-risk group, ${ }^{12}$ this could be explained by limited statistical power due to small sample size (UHR-P $n=12$, UHR-NP $n=23$ ). The study reported here thus complements and extends our previous findings in suggesting that those at ultra-high risk of psychosis exhibit bilateral superior temporal gyrus reductions. Finally, our cohort included a rather diverse population with psychotic symptoms, and the findings could be relevant to psychoses in general. Neurobiological similarities and differences between schizophrenia and other psychoses such as bipolar disorder remain controversial. ${ }^{38}$ Although no difference in superior temporal gyrus volume was found between participants who later developed schizophreniaspectrum disorders $(n=19)$ and those who developed affective psychosis $(n=10)$, further work will be required to clarify the diagnostic specificity of our findings in a larger sample.

\section{Future research}

Although we have demonstrated that superior temporal gyral changes are already present in people at ultra-high risk of psychosis, the most active and marked abnormalities of this brain region occur predominantly during the transition phase to active psychosis. This implies that this is a time of dynamic or even accelerated brain changes, which could be a target for intervention and preventive strategies.

Tsutomu Takahashi, MD, PhD, Melbourne Neuropsychiatry Centre, Department of Psychiatry, University of Melbourne, Australia, Department of Neuropsychiatry, University of Toyama, Japan, and CREST, JST, Japan; Stephen J. Wood, PhD, Melbourne Neuropsychiatry Centre, Department of Psychiatry, Univeristy of Melbourne; Alison R. Yung, MBBS, MD, MPM, FRANZCP, ORYGEN Research Centre, PACE Clinic and Department of Psychiatry, University of Melbourne; Mark walterfang, FRANZCP, ORYGEN Research Centre, PACE Clinic and Department of Psychiatry, University of Melbourne; Lisa J. Phillips, PhD, Department of Psychology, University of Melbourne; Bridget Soulsby, BSc, Melbourne Neuropsychiatry Centre, Department of Psychiatry, University of Melbourne; Yasuhiro Kawasaki, MD, PhD, Department of Neuropsychiatry, University of Toyama and CREST, JST; Patrick D. McGorry, MD, PhD, FRCP, FRANZCP, ORYGEN Research Centre, PACE Clinic and Department of Psychiatry, University of Melbourne; Michio Suzuki, MD, PhD, Department of Neuropsychiatry, University of Toyama and CREST, JST; Dennis Department of Neuropsychiatry, University of Toyama and CREST, JST; Dennis
Velakoulis, MBBS, FRANZCP, Christos Pantelis, MD, MRCPSych, FRANZCP, Melbourne Neuropsychiatry Centre, Department of Psychiatry, University of Melbourne

Correspondence: Dr Tsutomu Takahashi, Melbourne Neuropsychiatry Centre, c/o National Neuroscience Facility, 161 Barry Street, Carlton South, Victoria 3053 Australia. E-mail: tsutomu@med.u-toyama.ac.jp

First received 18 Jun 2009, final revision 7 Oct 2009, accepted 28 Oct 2009 


\section{Funding}

The study was supported by project grants from the National Health and Medical Research Council (NHMRC) (grants 145627, 145737, 970598, 981112, 970391), NHMRC programme grant 350241 and the Colonial Foundation. D.V. and S.J.W. were supported as research officers with funding from the NHMRC. M.W. was supported by a Stanley Research Centre grant. P.D.M. was supported by an NARSAD Distinguished Investigator Award. S.J.W. is currently supported by a Clinical Career Development Award from the NHMRC (359223) currently supported by a Clinical Career Development Award from the NHMRC (359223)
and an NARSAD Young Investigator Award. T.T. was supported to undertake this work by a grant-in-aid for scientific research (19591346) from the Japanese Society for the Promotion of Science, and a research grant $(17-2,18-6)$ for nervous and mental disorders from the Ministry of Health and Welfare, Japan.

\section{Acknowledgements}

Neuroimaging analysis was facilitated by the Neuropsychiatry Imaging Laboratory, managed by Ms Bridget Soulsby at the Melbourne Neuropsychiatry Centre, Australia, and supported by Neurosciences Victoria.

\section{References}

1 Hirayasu Y, Shenton ME, Salisbury DF, Dickey CC, Fischer IA, Mazzoni P, et al. Lower left temporal lobe MRI volumes in patients with first-episode schizophrenia compared with psychotic patients with first-episode affective disorder and normal subjects. Am J Psychiatry 1998; 155: 1384-91.

2 Keshavan MS, Haas GL, Kahn CE, Aguilar E, Dick EL, Schooler NR, et al. Superior temporal gyrus and the course of early schizophrenia: progressive, static, or reversible? J Psychiatr Res 1998; 32: 161-7.

3 Kim JJ, Crespo-Facorro B, Andreasen NC, O'Leary DS, Magnotta V, Nopoulos P. Morphology of the lateral superior temporal gyrus in neuroleptic naive patients with schizophrenia: relationship to symptoms. Schizophr Res 2003; 60: 173-81.

4 Hirayasu Y, McCarley RW, Salisbury DF, Tanaka S, Kwon JS, Frumin M, et al. Planum temporale and Heschl gyrus volume reduction in schizophrenia: a magnetic resonance imaging study of first-episode patients. Arch Gen Psychiatry 2000; 57: 692-9.

5 Kasai K, Shenton ME, Salisbury DF, Hirayasu Y, Onitsuka T, Spencer MH, et al Progressive decrease of left Heschl gyrus and planum temporale gray matter volume in first-episode schizophrenia: a longitudinal magnetic resonance imaging study. Arch Gen Psychiatry 2003; 60: 766-75.

6 Yung AR, Phillips LJ, Yuen HP, Francey SM, McFarlane CA, Hallgren M, et al. Psychosis prediction: 12-month follow up of a high-risk ('prodromal') group. Schizophr Res 2003; 60: 21-32.

7 Yung AR, Phillips L, Yuen HP, McGorry PD. Risk factors for psychosis in an ultra high-risk group: psychopathology and clinical features. Schizophr Res 2004; 67: 131-42.

8 Pantelis C, Velakoulis D, McGorry PD, Wood SJ, Suckling J, Phillips $\sqcup$, et al. Neuroanatomical abnormalities before and after onset of psychosis: a crosssectional and longitudinal MRI comparison. Lancet 2003; 361: 281-8.

9 Borgwardt SJ, Riecher-Rössler A, Dazzan P, Chitnis X, Aston J, Drewe M, et al. Regional gray matter volume abnormalities in the at risk mental state. Biol Psychiatry 2007; 61: 1148-56.

10 Crum WR, Griffin LD, Hill DL, Hawkes DJ. Zen and the art of medical image registration: correspondence, homology, and quality. Neuroimage 2003; 20 1425-37.

11 Rajarethinam R, Sahni S, Rosenberg DR, Keshavan MS. Reduced superior temporal gyrus volume in young offspring of patients with schizophrenia. Am J Psychiatry 2004; 161: 1121-4.

12 Takahashi T, Wood SJ, Yung A, Soulsby B, McGorry PD, Suzuki M, et al. Progressive gray matter reduction of the superior temporal gyrus during transition to psychosis. Arch Gen Psychiatry 2009; 66: 366-76.

13 Nelson $\mathrm{HE}$, O'Connell A. Dementia: the estimation of premorbid intelligence levels using the New Adult Reading Test. Cortex 1978; 14: 234-44.

14 Yung AR, Phillips LJ, McGorry PD. Treating Schizophrenia in the Prodromal Phase. Taylor \& Francis, 2004.

15 Rhoades HM, Overall JE. The semistructured BPRS interview and rating guide Psychopharmacol Bull 1988; 24: 101-4.
16 Andreasen NC. The Scale for the Assessment of Negative Symptoms (SANS) University of lowa, 1983.

17 Maxwell ME. Family Interview for Genetic Studies (FIGS): A Manual for FIGS. Clinical Neurogentics Branch, Intramural Research Program, National Institute of Mental Health, 1992.

18 Yung AR, Yuen HP, McGorry PD, Phillips LJ, Kelly D, Dell'Olio M, et al. Mapping the onset of psychosis: the Comprehensive Assessment of At-Risk Mental States. Aust N Z J Psychiatry 2005; 39: 964-71.

19 First MB, Spitzer RL, Gibbon M, Williams JB. Structured Clinical Interview for DSM-IV Axis I Disorders. American Psychiatric Press, 1997.

20 American Psychiatric Association. Quick Reference to the Diagnostic Criteria From DSM-IV. APA, 1994.

21 Eritaia J, Wood SJ, Stuart GW, Bridle N, Dudgeon P, Maruff $P$, et al. An optimized method for estimating intracranial volume from magnetic resonance images. Magn Reson Med 2000; 44: 973-7.

22 Takahashi T, Suzuki M, Zhou SY, Tanino R, Hagino $H$, Kawasaki $Y$, et al. Morphologic alterations of the parcellated superior temporal gyrus in schizophrenia spectrum. Schizophr Res 2006; 83: 131-43.

23 Meisenzahl EM, Koutsouleris N, Bottlender R, Scheuerecker J, Jäger M, Teipel $\mathrm{SJ}$, et al. Structural brain alterations in subjects at high-risk of psychosis: a voxel-based morphometric study. Schizophr Res 2008; 102: 150-62.

24 Takahashi T, Wood SJ, Soulsby B, Kawasaki Y, McGorry PD, Suzuki M, et al. An MRI study of the superior temporal subregions in first-episode patients with various psychotic disorders. Schizophr Res 2009; 113: 158-66.

25 Borgwardt SJ, McGuire PK, Aston J, Berger G, Dazzan P, Gschwandtner U, et al. Structural brain abnormalities in individuals with an at-risk mental state who later develop psychosis. Br J Psychiatry 2007; 191 (suppl 51): s69-75.

26 Pantelis C, Yucel M, Wood SJ, Velakoulis D, Sun D, Berger G, et al. Structural brain imaging evidence for multiple pathological processes at different stages of brain development in schizophrenia. Schizophr Bull 2005; 31 672-96.

27 Job DE, Whalley HC, McConnell S, Glabus M, Johnstone EC, Lawrie SM. Voxelbased morphometry of grey matter densities in subjects at high risk of schizophrenia. Schizophr Res 2003; 64: 1-13.

28 Pantelis C, Velakoulis D, Wood SJ, Yücel M, Yung AR, Phillips L, et al. Neuroimaging and emerging psychotic disorders: the Melbourne ultra-high risk studies. Int Rev Psychiatry 2007; 19: 371-81.

29 Barta PE, Pearlson GD, Powers RE, Richards SS, Tune LE. Auditory hallucinations and smaller superior temporal gyral volume in schizophrenia. Am J Psychiatry 1990; 147: 1457-62.

30 Shenton ME, Kikinis R, Jolesz FA, Pollak SD, LeMay M, Wible CG, et al. Abnormalities of the left temporal lobe and thought disorder in schizophrenia. A quantitative magnetic resonance imaging study. N Engl J Med 1992; 327: 604-12.

31 Gallagher $\mathrm{HL}$, Frith CD. Functional imaging of 'theory of mind'. Trends Cogn Sci 2003; 7: 77-83.

32 McGorry PD, Hickie IB, Yung AR, Pantelis C, Jackson HJ. Clinical staging of psychiatric disorders: a heuristic framework for choosing earlier, safer and more effective interventions. Aust N Z J Psychiatry 2006; 40: 616-22.

33 Garner B, Pariante CM, Wood SJ, Velakoulis D, Phillips L, Soulsby B, et al. Pituitary volume predicts future transition to psychosis in individuals at ultrahigh risk of developing psychosis. Biol Psychiatry 2005; 58: 417-23.

34 Fornito A, Yung AR, Wood SJ, Phillips LJ, Nelson B, Cotton S, et al. Anatomic abnormalities of the anterior cingulate cortex before psychosis onset: an MRI study of ultra-high-risk individuals. Biol Psychiatry 2008; 64: 758-65.

35 Walterfang M, Yung A, Wood AG, Reutens DC, Phillips L, Wood SJ, et al. Corpus callosum shape alterations in individuals prior to the onset of psychosis. Schizophr Res 2008; 103: 1-10.

36 Scherk H, Falkai P. Effects of antipsychotics on brain structure. Curr Opin Psychiatry 2006; 19: 145-50.

37 Yücel M, Solowij N, Respondek C, Whittle S, Fornito A, Pantelis C, et al Regional brain abnormalities associated with long-term heavy cannabis use. Arch Gen Psychiatry 2008; 65: 694-701.

38 Maier W, Zobel A, Wagner M. Schizophrenia and bipolar disorder: differences and overlaps. Curr Opin Psychiatry 2006; 19: 165-70. 